\title{
Percepción de las enfermeras sobre la educación en accesos vasculares en hemodiálisis. Estudio fenomenológico
}

\author{
Javier Alonso Bula-Romero, Luz Miriam Calderón-Quintana, Carlos Darío Oquendo-Martínez
}

Facultad Ciencias de la Salud. Universidad de Córdoba. Montería. Colombia

Como citar este artículo:

Bula-Romero JA, Calderón-Quintana LM, Oquendo-Martínez CD. Percepción de las enfermeras sobre la educación en accesos vasculares en hemodiálisis. Estudio fenomenológico.

Enferm Nefrol. 2020 Ene-Mar;23(1):45-52

\section{Resumen}

Introducción: La Enfermedad Renal Crónica (ERC), representa un problema de salud pública que genera un desafío para quien la padece y quienes intervienen en la prevención, el tratamiento y rehabilitación de esta condición de salud. En ese sentido, es importante tener en cuenta que la educación para el autocuidado juega un papel trascendental al momento de generar cambios en la calidad de vida de este grupo poblacional.

Objetivo: El Objetivo de este estudio fue describir los elementos que orientan la educación para el autocuidado de los pacientes con accesos vasculares en tratamiento de hemodiálisis desde; la perspectiva de enfermería.

Materiales y métodos: Estudio descriptivo cualitativo, de diseño narrativo con aproximación fenomenológica; desarrollado a partir de la técnica de análisis de contenido. Los sujetos de estudio fueron 10 profesionales de enfermería que laboran en Unidades Renales de Montería-Córdoba (Colombia). La información fue recolectada a través de dos tipos de técnicas, una de ellas fue la entrevista semiestructurada y la otra los diarios de campo.

Resultados: De esta investigación surgieron tres unidades de análisis: el cuidado de enfermería como sistema de apoyo educativo para el autocuidado; la relación empática entre la enfermera y el paciente como elemento

\section{Correspondencia:}

Javier Alonso Bula Romero

E-mail: Javierbula@correo.unicordoba.edu.co facilitador en la continuidad del autocuidado y el trabajo multidisciplinar como estrategia para el mantenimiento de la salud y el bienestar del paciente.

Discusión: La literatura es clara en demostrar que el cuidado de enfermería, la relación empática y el trabajo multidisciplinar, facilitan la adherencia de los pacientes con enfermedad renal crónica al tratamiento dialítico.

Conclusiones: La educación para el autocuidado es un elemento fundamental para el manejo del paciente con acceso vascular, y surge como una herramienta para el conocimiento y el desarrollo de habilidades de autocuidado en el paciente con enfermedad renal crónica.

PALABRAS CLAVE: educación; autocuidado; cuidado de Enfermería y hemodiálisis.

\section{Nurses' perception of education in vascular accesses in hemodialysis. Phenomenological study.}

\section{Abstract}

Introduction: Chronic Kidney Disease (CKD) represents a public health problem that creates a challenge for patients and those involved in the prevention, treatment and rehabilitation of this health condition. In that sense, it is important to keep in mind that education in selfcare is decisive to generate changes in the quality of life of this population group. 
Objective: The objective of this study was to describe the elements that guide education in the self-care of patients with vascular access in hemodialysis treatment, from the nursing perspective.

Materials and Method: Qualitative descriptive study, of narrative design with phenomenological approach; using the content analysis technique. The study subjects were 10 nursing professionals working in Renal Units of Montería-Córdoba (Colombia). The information was collected through two types of techniques, semistructured interview and field diaries.

Results: Three units of meaning emerged from this research: nursing care as an educational support system for self-care; the empathic relationship between the nurse and the patient as a facilitating element in the continuity of self-care; and multidisciplinary work as a strategy for the maintenance of the health and wellbeing of the patient.

Discussion: The evidence is clear in demonstrating that nursing care, empathic relationships and multidisciplinary work facilitate the adherence of patients with chronic kidney disease to dialysis.

Conclusions: Education for self-care is a fundamental element for the management of patients with vascular access, and emerges as a tool for the knowledge and development of self-care skills in patients with chronic kidney disease.

KEYWORDS: education; self-care; nursing care; hemodialysis.

\section{Introducción}

La Enfermedad Renal Crónica (ERC), representa un problema de salud pública a nivel mundial que afecta cerca del $10 \%$ de la población en general ${ }^{1}$. En América Latina, según reportes de la Sociedad Latinoamericana de Nefrología e Hipertensión (SLANH)², para el 2017, cerca de 66,8 por 100.000 habitantes, iniciaron terapia de reemplazo renal con hemodiálisis, diálisis peritoneal y trasplantes de riñón, porque padecían insuficiencia renal crónica.

En Colombia, según el informe de la cuenta de enfermedades de Alto Costo del 2017, se estimó que las terapias de reemplazo renal generaron entre el $2,5 \%$ y el $3 \%$ del gasto del presupuesto del Sistema Nacional de Salud y más del $4 \%$ en la atención especializada 3 .

El presente estudio toma como base teórica la propuesta de Dorotea Orem, que define el autocuidado como una actividad aprendida por los individuos y orientada a situaciones concretas de la vida ${ }^{4}$. Dorothea Orem, destaca el autocuidado como una herramienta de vital importancia en el abordaje de los seres humanos, en especial de aquellos que por condiciones de salud ven afectada su cotidianidad. La teoría de Orem, concibe el autocuidado como acciones que los individuos hacen por su propio bien, para mantener la vida, la salud y su bienestar ${ }^{5}$.

Orem reconoce la capacidad del ser humano para reflexionar y poner en práctica los mecanismos para identificar necesidades y suplirlas mediante el autocuidado, consideración que permite empoderar al individuo y tomar la decisión personal de cuidar de su propia saludt.

Conocer desde la perspectiva de enfermería los elementos que orientan la educación para el autocuidado en pacientes con accesos vasculares, permitirá reevaluar las estrategias para su abordaje en la práctica asistencial; en este sentido, el cuidado de enfermería estará centrado en el paciente, favoreciendo de esta manera su autocuidado, mejorando su salud y bienestar? ${ }^{7}$.

El presente estudio planteó como objetivo general describir los elementos que orientan la educación para el autocuidado de los pacientes con accesos vasculares en tratamiento de hemodiálisis desde la perspectiva de enfermería.

\section{Material y Método}

Estudio descriptivo cualitativo, de diseño narrativo con aproximación fenomenológica; desarrollado a partir de la técnica de análisis de contenido. Esta fue definida como un conjunto de técnicas de análisis que utiliza procedimientos sistemáticos para la descripción del contenido manifiesto o latente presente en la comunicación, permitiendo de esta manera, la inferencia de conocimientos relacionados con las condiciones de producción y recepción de los mensajes de comunicación; propuesta por Laurence Bardin ${ }^{8}$.

En los diseños narrativos el investigador recolecta información de las experiencias de un determinado grupo personas, para describirlas y analizarlas ${ }^{9}$. Esta investi- 
Tabla 1. Características sociodemográficas de los participantes de estudio.

\begin{tabular}{|c|c|c|c|c|c|c|}
\hline NOMBRE & EDAD & SEXO & $\begin{array}{l}\text { ESPECIALIDAD EN } \\
\text { ENFERMERÍA } \\
\text { NEFROLÓGICA }\end{array}$ & $\begin{array}{c}\text { TIEMPO DE } \\
\text { EXPERIENCIA } \\
\text { EN LA UNIDAD RENAL }\end{array}$ & $\begin{array}{l}\text { CONDICIÓN } \\
\text { SOCIAL }\end{array}$ & $\begin{array}{c}\text { AREA DE } \\
\text { RESIDENCIA }\end{array}$ \\
\hline Enfermera 1 (El) & 30 & Masculino & Sí & 5 años & Unión Libre & Urbana \\
\hline Enfermera 2 (E2) & 38 & Femenino & Sí & 5 años & Casada & Urbana \\
\hline Enfermera 3 (E3) & 40 & Femenino & No & 10 años & Casada & Urbana \\
\hline Enfermera 4 (E4) & 28 & Masculino & No & 8 años & Unión Libre & Urbana \\
\hline Enfermera 5 (E5) & 30 & Masculino & No & 9 años & Unión Libre & Urbana \\
\hline Enfermera 6 (E6) & 34 & Masculino & No & 10 años & Casado & Urbana \\
\hline Enfermera 7 (E7) & 29 & Masculino & Sí & 6 años & Unión Libre & Urbana \\
\hline Enfermera 8 (E8) & 27 & Masculino & No & 5 años & Soltero & Urbana \\
\hline Enfermera 9 (E9) & 26 & Masculino & No & 5 años & Soltero & Urbana \\
\hline Enfermera 10 (E10) & 32 & Femenino & No & 7 años & Unión Libre & Urbana \\
\hline
\end{tabular}

Fuente. Entrevistas del estudio: educación para el autocuidado en pacientes con accesos vasculares en tratamiento de hemodiálisis: una mirada desde enfermería.

gación indagó precisamente las experiencias de un grupo de enfermeros que trabajan en las unidades renales de la ciudad de Montería-Córdoba, con el fin de describir y analizar los elementos que orientan la educación para el autocuidado en pacientes con accesos vasculares en tratamiento de hemodiálisis.

Para la muestra de estudio se tuvo en cuenta los criterios de suficiencia y adecuación de los datos, propuestos por Denzin y Lincoln ${ }^{10}$, los cuales hacen referencia a la calidad de información recolectada, antes que al número de sujetos entrevistados. La suficiencia de la información se alcanzó cuando las respuestas de las participantes tenían elementos comunes, es decir cuando repetían la misma información y no se ofrecían aportes nuevos al estudio.

La información se obtuvo a través de una entrevista semiestructurada, conformada por 8 preguntas orientadoras abiertas que guiaron el curso de la conversación, dando matices significativos al objeto de estudio, que a su vez, se relacionaban el objetivo propuesto para esta investigación. A medida que compartían sus experiencias, permitían a los investigadores identificar situaciones que facilitaban la comprensión del fenómeno de estudio.

Para la construcción del guion de la entrevista semiestructurada, se requirió inicialmente realizar una prueba piloto: que llevó a cabo 4 entrevistas, que permitieron refinar el proceso de recolección de la información, y estructurar un conjunto de preguntas abiertas y cerradas, orientadas a alcanzar el propósito de estudio.
El tiempo de duración de las entrevistas fue entre 25 a 30 minutos, los investigadores permanecieron en escucha activa a través del contacto visual con los participantes mientras se realizaban grabaciones de voz con dispositivos electrónicos que posteriormente fueron transcritas fielmente a través de un software de uso libre para transcripción de audios denominado oTranscribe ${ }^{11}$.

\section{Participantes de estudio}

Una vez aceptada la invitación, se explicó el objetivo general de la investigación y los procedimientos contemplados para el análisis de la información. Una vez finalizado, se procedió a la firma del consentimiento para participar en el estudio.

En esta investigación, se realizó un muestreo intencional; la lógica y el poder de las muestras intencionales reside en identificar los casos ricos en información, para estudiarlos en profundidad; los casos ricos en información, son aquellos que aportan elementos claves o centrales relacionados con el propósito de la investigación.

Participaron 10 enfermeros y enfermeras que laboran en las diferentes unidades renales del municipio de Montería-Córdoba (Colombia). Estas Unidades Renales se encontraban ubicadas en zona céntrica de la Ciudad de Montería y eran responsables de los tratamientos dialíticos en usuarios con Enfermedad Renal Crónica afiliados al régimen subsidiado y contributivo del Sistema General de Seguridad Social en Salud del Departamento de Córdoba. En la Tabla 1 se describen las características sociodemográficas de los participantes en el estudio. 
La presente investigación se respaldó en los parámetros éticos para estudios con seres humanos contenidos en la Resolución 008430 de 1993 del Ministerio de Salud de Colombia ${ }^{12}$, en la Ley 911 de $2004^{13}$ y los requerimientos básicos para evaluar aspectos éticos en investigación, propuestos por Ezekiel Emanue ${ }^{14}$. Asimismo, este proyecto de investigación fue aprobado por el comité de ética de la Facultad Ciencias de la Salud de la Universidad de Córdoba-Colombia.

En todas las fases de la investigación se mantuvo la fidelidad al pensamiento y a las expresiones de los participantes de estudio, se realizaron transcripciones textuales de las grabaciones, así mismo, se solicitó autorización para ser contactados nuevamente en caso de requerir ampliar alguna información o revisar conjuntamente las transcripciones de las entrevistas y realizar devolución y validación de los resultados.

\section{Planificación del análisis de contenido}

El análisis de la información se realizó de manera sistemática a través de la recolección-análisis-recolección y análisis de los datos, hasta alcanzar la saturación teórica. Esta investigación se realizó a partir de la técnica de análisis de contenido, que consiste en ordenar la información, codificarla, categorizar los códigos y definir los temas o las unidades de análisis que derivan directamente del texto.

Para la depuración de la información se organizaron archivos independientes, asignando un código alfa numérico a cada archivo identificando el número de entrevista y las letras iniciales del primer nombre y los apellidos de los participantes de estudio. Esto permitió la organización del documento de manera cronológica, identificando las narraciones de cada uno de ellos; así mismo, cada archivo contenía las transcripciones de las entrevistas y los diarios de campo de la investigación, insumo importante para el análisis de contenido propuesto por Bardin.

\section{Codificación de la información}

La información se organizó en una matriz de codificación para revisar minuciosamente cada texto de las entrevistas con el fin de encontrar las palabras o frases que permitieron identificar los códigos. Este paso de codificación consistió en hallar palabras o frases dentro de los datos que permitieron identificar los elementos subyacentes para ser analizados, organizar la información y realizar anotaciones de datos importantes que luego serán empleados en el análisis.
Se definieron las categorías y las subcategorías teniendo en cuenta la homogeneidad interna (todos los datos reflejan la categoría) y la homogeneidad externa (muestra la relación entre categorías y subcategorías) finalmente, se realizó la descripción y análisis de las categorías emergentes utilizando la literatura disponible para soportar los hallazgos encontrados que posteriormente fueron ampliados en la discusión de los resultados.

Los pasos que se llevaron a cabo para la planificación del análisis de contenido contempló las siguientes etapas: establecimiento del corpus donde se delimitó el número de entrevistas necesarias por participante, hasta lograr la saturación de la información (en total se realizaron 20 entrevistas; dos por cada participante).

La preparación del material donde cada texto fue resultado de las transcripciones fieles de las entrevistas realizadas y la etapa de análisis donde cada entrevista fue organizada buscando la alineación, el estilo y los elementos atípicos del discurso; en esta investigación, fueron las expresiones verbales el fundamento del análisis, las cuales se transcribieron textualmente con el fin de mantener objetividad por parte de los investigadores, y de esta manera facilitar su manejo y análisis.

Los códigos y categorías se nombraron con las mismas palabras del discurso de los participantes con el ánimo de mantener la fidelidad al texto analizado y la rigurosidad de la metodología de análisis.

\section{Resultados}

Emergieron tres unidades de análisis que permiten describir y comprender los elementos que orientan la educación para el autocuidado en los pacientes con accesos vasculares en tratamiento de hemodiálisis, representadas en las siguientes categorías: el cuidado de enfermería como sistema de apoyo educativo para el autocuidado, la relación empática entre la enfermera y el paciente como elemento facilitador en la continuidad del autocuidado y el trabajo multidisciplinar como estrategia para el mantenimiento de la salud y el bienestar del paciente.

Desde el cuidado de enfermería, el apoyo educativo es visto como el eje central para potencializar el autocuidado en pacientes con accesos vasculares en tratamiento de hemodiálisis; como lo refiere Acosta ${ }^{15}$, quien manifiesta que la educación es un proceso continuo de enseñanza donde la enfermera utiliza las habilidades, 
conocimientos y destrezas que ha adquirido a través de su práctica profesional para favorecer comportamientos de autocuidado en pacientes con enfermedad renal crónica.

Es importante tener en cuenta que el conocimiento que tienen las personas de su enfermedad se puede fortalecer con la educación; como se manifiesta en las siguientes declaraciones:

"... ¡La educación es un elemento primordial para el autocuidado! como enfermeros debemos enseñarle al paciente qué tiene que hacer y por qué debe hacerlo!..."

E1- 30 años

"... ¡Aquí se hace mucha educación al paciente con enfermedad renal en tratamiento de hemodiálisis! ;Sobre todo, los cuidados que éste debe tener con su acceso vascular!"

E2- 38 años

"... ¡Hay que ser constante con la educación del paciente! aún si este es un paciente renuente, o tenga un insight negativo de su enfermedad; con estos pacientes, hay que ser más constantes..."

E3-40 años

"... ¡Algunos pacientes son muy receptivos! ¡Otros no aceptan que están enfermos!, entonces uno debe ayudarlos en ese sentido..."

E9-26 años

Claramente se puede evidenciar que la fortaleza del personal de enfermería es el rol que este ejerce como educador, generalmente es quien se encarga de guiar, apoyar y proporcionar un entorno que estimula el autocuidado del paciente ${ }^{16}$. De igual forma Enfermería representa un sistema de apoyo educativo, donde se establece que el paciente es quien realiza el autocuidado; mientras la enfermera es quien formula las líneas de conducta necesarias para responder a cada necesidad identificada ${ }^{17}$.

La relación empática entre la enfermera y el paciente como elemento facilitador en la continuidad del autocuidado

La relación enfermera-paciente se presenta por las interacciones que se dan entre estos dos sujetos, donde cada uno de ellos tiene una influencia recíproca en el otro. Según Salázar y Martínez ${ }^{18}$, cuando una enfermera se esfuerza en conocer a su paciente, acierta en reconocer sus necesidades y logra comprender los significados que este otorga a su experiencia de salud, puede brindar un cuidado integral y acorde a lo esperado.

"... Uno se esfuerza mucho por ellos, para lograr su mejoría y favorecer una mejor calidad de vida..."

E4-28 años

"... Todo depende de la empatía que uno tenga con el paciente. ¡Debemos ser muy empáticos con él y tratar de manejar a los pacientes difíciles!..."

E5-30 años

"... Pienso que todo depende de la empatía que uno tenga con el paciente!..."

E10-32 años

Ser empático en enfermería significa "percibir a profundidad la razón especial y única que cada enfermo tiene de su enfermedad, cómo la entiende, lo que este siente, e incluso la aceptación de la misma; en pocas palabras, ponerse en el lugar del otro"119. Por ello, La enfermera debe tener la habilidad de percibir los sentimientos que tiene el paciente y ponerse en su lugar para comprender su situación.

El trabajo multidisciplinar como estrategia para el mantenimiento de la salud y el bienestar del paciente Cuando un paciente ingresa a una sala de hemodiálisis, generalmente es abordado por el profesional de enfermería, quien es el encargado de identificar sus necesidades de cuidado, direccionándolas a otros integrantes del equipo interdisciplinario cuando no hay respuesta 0 adherencia al tratamiento ${ }^{20}$.

"...Nosotros siempre vamos a estar ahí, pendiente a ellos, pues de nosotros depende el éxito de la terapia..."

E6-34 años

¡Cuando el paciente es renuente al tratamiento, buscamos ayuda; nosotros siempre trabajamos en equipo, todos somos un equipo y nuestro objetivo es manejar integralmente al paciente!

E7-29 años

"... Entre todos existe una conexión, si yo valoro mi paciente y sé que no está bien..., pido una segunda valoración, para eso estamos! somos un equipo de trabajo que siempre busca la salud y el bienestar del paciente..." E8-27 años 
Como se puede evidenciar, el trabajo multidisciplinar como estrategia para el mantenimiento de la salud, influye de manera positiva en el abordaje del paciente renal. Urzúa et $\mathrm{al}^{21}$, afirma que la relación que existe entre un proveedor de salud y un paciente es determinante para llevar a cabo la adherencia a las recomendaciones prescritas; esta relación de confianza entre el equipo interdisciplinar y la persona enferma puede ayudar a mejorar las condiciones de salud y bienestar del paciente.

\section{Discusión}

La educación se considera un factor importante en la promoción y la reducción o exacerbación de las condiciones de co-morbilidad en los pacientes con insuficiencia renal crónica ${ }^{22}$. Sin embargo, Guerra et al23, afirman que es necesario realizar más de una intervención educativa para lograr con éxito la adherencia de un paciente al tratamiento. De otra parte, Ramos ${ }^{24}$, plantea que la educación es una herramienta que permite mejorar, aumentar y mantener la adherencia de los pacientes en tratamiento de hemodiálisis, facilitando su adaptación al tratamiento.

La experiencia de vivir con enfermedad renal crónica genera un impacto en la vida de quienes la padecen. EI desconocimiento de la enfermedad y por consiguiente del tratamiento, puede generar sentimientos y comportamientos que pueden afectar la capacidad que estos individuos tienen para cuidar de su propia saludd ${ }^{25}$; es allí donde el autocuidado puede verse comprometido, y el profesional de enfermería está llamado a potenciar o apoyar las experiencias de salud de estas personas, acompañándolos en su proceso, con el fin de lograr que ellos afronten esta situación para facilitar su calidad de vida ${ }^{26}$.

En muchas ocasiones los pacientes sometidos a hemodiálisis requieren de acompañamiento y apoyo emocional para afrontar los cambios generados por la enfermedad ${ }^{27}$; en este sentido, el personal de enfermería puede apoyarse de la familia del paciente para que éste pueda lograr una mayor adaptación, asimilación y comprensión del proceso salud-enfermedad, permitiendo que asuma posturas positivas en torno al tratamiento de la hemodiálisis ${ }^{28}$.

Existen pacientes renuentes a las recomendaciones dadas por el profesional de salud. Ante esta situación, una segunda opción es intervenir a la familia como elemento clave para fortalecer el autocuidado del paciente ${ }^{29}$, en este momento el ambiente familiar se vuelve importante porque la enfermera puede intervenir al núcleo familiar indicando las ventajas del autocuidado.
Es evidente que la enfermera apoya constantemente al paciente durante este proceso, dándole herramientas que fortalezcan su autocuidado; en este sentido, Rojas et al ${ }^{30}$, sostienen que el profesional de enfermería asume mayor responsabilidad cuando evidencia en el paciente ciertas debilidades, evitando posibles complicaciones que pueden repercutir directamente en la funcionalidad del acceso vascular.

Una de las barreras que enfrentan el personal de enfermería es la actitud del paciente frente a lo desconocido. Por esa razón Muñoz et al ${ }^{31}$, manifiestan que para llegar a generar empatía es necesario comenzar por identificarnos con la persona, con sus sentimientos y emociones, a través de la comunicación, así podremos comprender y percibir los significados que éste otorga a su experiencia y cuáles son las verdaderas necesidades para poder brindar los cuidados necesarios.

En las salas de hemodiálisis, el acercamiento enfermero-paciente se logra con la empatía, en estas salas todos se conocen, se habla abiertamente, se maneja un lenguaje coloquial y se tiene en cuenta sus opiniones ${ }^{32}$. Para López, estos espacios han fortalecido la construcción de una relación más cercana entre la enfermera y los pacientes de las unidades renales ${ }^{33}$.

La interacción que se da en las salas de hemodiálisis crea un vínculo entre el profesional de enfermería y el paciente; es aquí donde el éxito de la relación terapéutica cobra importancia; por lo tanto, para Sanz et a ${ }^{34}$, la clave para que este proceso tenga éxito, es el uso de la comunicación asertiva.

Se puede afirmar que la educación para el autocuidado podría generar grandes cambios en el abordaje de un paciente renal, para ello es indispensable no sólo poner en juego todos y cada uno de los elementos aquí dilucidados, sino que se hace necesario sumar esfuerzos que apunten a fortalecer el rol de enfermería en cada uno de los actos de cuidado ${ }^{35}$.

A partir de las categorías emergentes de este estudio, se consolida la oportunidad que tienen los profesionales de Enfermería para fortalecer el autocuidado de un paciente con enfermedad renal a través de la interacción terapéutica y una práctica multidisciplinar, definiendo la educación como eje central para el fortalecimiento de estos procesos.

La educación es un elemento clave para el abordaje del autocuidado y surge como una herramienta fundamental 
que contribuye en la adquisición de conocimientos, habilidades y patrones de comportamiento en el paciente renal en tratamiento de hemodiálisis mejorando su calidad de vida y bienestar.

El vínculo entre la enfermera y el paciente permite el fortalecimiento de la relación terapéutica, favoreciendo líneas de acción que facilitan el uso de intervenciones para guiar los procesos de autocuidado en el paciente renal con acceso vascular.

La interacción con el equipo interdisciplinario genera acciones que permiten la continuidad del cuidado, involucrando todas las disciplinas que intervienen en el abordaje de un paciente renal, así mismo redirecciona la práctica del cuidado de Enfermería desde una visión integral e integrada.

Recibido: 15-11-19

Revisado: 05-12-19

Modificado: 22-12-19

Aceptado: 15-01-20

\section{Bibliografía}

1. Antón-Pérez G, Pérez-Borges $P$, Alonso-Alman $F$, Vega-Díaz N. Accesos vasculares en hemodiálisis: un reto por conseguir. Nefrología 2012; 32(1):103-7.

2. Lopera-Medina MM. La enfermedad renal crónica en Colombia: necesidades en salud y respuesta del Sistema General de Seguridad Social en Salud. Rev. Gerenc. Polít. Salud. 2016;15(30):212-33.

3. Ministerio de Salud y Protección Social. Cuenta de alto costo. 2017 [Documento en línea] Disponible desde internet en https://cuentadealtocosto.org/ site/index.php/component/content/article/9-patologias/35-enfermedad-renal-cronica-erc/?template=cuentadealtocostocontenido.

4. Prado Solar LA, González Reguera M, Paz Gómez N, Romero Borges K. La teoría Déficit de autocuidado:

Dorothea Orem punto de partida para calidad en la atención. Rev. Med. Electron. 2014;36(6):835-45.

5. Hernández M. Autocuidado y promoción de la salud en el ámbito laboral. Revista salud bosque. 2016;5(2): 79-88.

6. Arredondo A, Arango S. Práctica de autocuidado de los pacientes en hemodiálisis con los accesos vasculares en una unidad renal de la ciudad de montería 2017. Trabajo de grado Enfermero. Montería- Córdoba. Universidad de Córdoba. Facultad Ciencias de la Salud. Programa de Enfermería. 2017;16.

7. Pereira J, Boada et al. Diálisis y hemodiálisis. Una revisión actual según la evidencia. Revista Argentina de Nefrología. 2017;15(2):1-19.

8. Noguero F. Metodología participativa en la enseñanza universitaria. Madrid: Narcea Ediciones; 2005.

9. Salgado Levano AC. Investigación cualitativa: diseños, evaluación del rigor metodológico y retos. Liberabit. 2007;13(13):71-8.

10. González R. La investigación en la práctica educativa: Guía metodológica de investigación para el diagnóstico y evaluación en los centros docentes. Madrid: Ministerio de Educación y Ciencia; 2007.

11. Bentley E. Otranscribe. Herramienta web gratuita para la transcripción de archivos de audios. [Consultado 02 jul 2019]. Disponible en: https://otranscribe. $\mathrm{com} /$.

12. Ministerio de Salud. República de Colombia. Resolución 008430 de 1993 (octubre 4), por la cual se establecen las normas científicas, técnicas y administrativas para la investigación en salud.

13. República de Colombia. Tribunal Nacional Ético de Enfermería, TNEE. Ley 911 de 2004, octubre 5.

14. Emanuel EJ, Wendler D, Grady C. What makes clinical research ethical? JAMA. 2000;283(20):2701-11.

15. Acosta M. Explorando la teoría general de enfermería de Orem. Rev. cient. Soc. Esp. Enferm. Neurol. 2011;10(3):163-67.

16. Rivera L. Autocuidado y capacidad de agencia de autocuidado. Av Enfermer 2006;24(2):91-8. 
17. Naranjo Hernández Y, Concepción Pacheco JA, Rodríguez Larreynaga M. La teoría Déficit de autocuidado: Dorothea Elizabeth Orem. Gac Méd Espirit. 2017;19 (3):89-100.

18. Salazar Á, Martínez C. Un sobrevuelo por algunas teorías donde la interacción enfermería-paciente es el núcleo del cuidado. Av Enfermer 2008;26(2):107-15.

19. Müggenburg Rodríguez Vigil MC, Riveros-Rosas A. Interacción enfermera-paciente y su repercusión en el cuidado hospitalario: Parte II. Enferm. Univ 2012;9(2):6-13.

20. Galera-Fernández A, Martínez-de Merlo MT, Ochando-García A. Accesos vasculares para hemodiálisis: cuidados de enfermería. Angiología. 2005;57(Supl 2):S159-68.

21. Urzúa A, Pavlov R, Cortés R, Pino V. Factores Psicosociales Relacionados con la Calidad de Vida en Salud en Pacientes Hemodializados. Ter. psicol. 2011;29 (1):135-40.

22. Vanegas B. Experiencias de profesionales de enfermería en el cuidado psicoemocional a pacientes en la unidad de cuidados intensivos 2006. Rev. Colom. Enferm. 2016;3(3):21-31.

23. Guerra Guerrero VT, Díaz Mujica AE, Vidal Albornoz K. La educación como estrategia para mejorar la adherencia de los pacientes en terapia dialítica. Rev Cubana Enfermer 2010;26(2):52-62.

24. Ramos Morales LE. La adherencia al tratamiento en las enfermedades crónicas. Rev Cubana Angiol Cir Vasc. 2015;16(2):175-89.

25. Gómez Vahos LE, Muriel Muñoz LE, Londoño-Vásquez DA. Prácticas evaluativas en la escuela. Una ruta pedagógica hacia la construcción de aprendizajes significativos. Revista Aletheia 2019;11(1):37-68.

26. Valderrama Sanabria ML, Peña Pita AP, Clavijo Álvarez LM. Narrativa: el estudiante de enfermería aprendiendo el arte de cuidar. Rev Cuid 2017;8(1):1488-98.
27. Sánchez Raja E, López Lara $P$, Honrubia Pérez M. Intervenciones enfermeras en cuidados sanitarios de sexualidad para mujeres con lesión medular lumbar en Cataluña. International Journal of Developmental and Educational Psychology 2018;3(1):109-17.

28. Cárdenas U, Alessandra G. Autocuidado del paciente adulto en tratamiento de hemodiálisis. Hospital Nacional Dos de Mayo diciembre. 2016:1-90.

29. Hernández NE, Moreno CM, Barragán JA. Necesidades de cuidado de la díada cuidador-persona: expectativa de cambio en intervenciones de enfermería. Rev Cuid 2014;5(2):748-56.

30. Rojas Estrada JJ, Alfonso Sat F, Castelo Villalón X, Semanat Vaillant D. Calidad del modelo de gestión actual del acceso vascular en los pacientes en hemodiálisis. Rev Cubana Med 2012;51(2):106-16.

31. Muñoz Devesa A, Morales Moreno I, Bermejo Higuera JC, Galán González Serna JM. La Relación de ayuda en Enfermería. Index Enferm 2014;23(4):229-33.

32. Segura Barrios IM, Barrera Ortiz L. Un Ilamado a Enfermería para responder al cuidado de la salud de las personas en situación de enfermedad crónica por su impacto en su calidad de vida. Salud Uninorte.2016; 32(2):228-43.

33. López González A, Díaz Rodríguez L, Ornosa Agra C. La unidad de diálisis, ¿un entorno accesible? Enfermer Nefrol 2012;15(2):121-27.

34. Sanz M, Garrido L, Caro C. Factores que influyen en la satisfacción del paciente de diálisis con enfermería. Enfermer Nefrol 2017;20(1):66-75.

35. Triana Restrepo MC. Efecto del vínculo empático enfermera-paciente sobre el nivel de ansiedad del paciente adulto en la unidad de cuidado intensivo. Tesis Doctoral. Bogotá: Universidad Nacional de Colombia;2016.

Este artículo se distribuye bajo una Licencia Creative Commons Atribución-NoComercial 4.0 Internacional. https://creativecommons.org/licenses/by-nc/4.0/ 